\title{
Precision Oscillation Measurements with Next Generation Reactor Neutrinos
}

\author{
A. Garfagnini* \\ Università di Padova and INFN-Padova \\ E-mail: alberto.garfagnini@pd.infn.it \\ on behalf of the JUNO Collaboration
}

Precise measurements of the $\theta_{13}$ neutrino oscillation parameter by the Daya Bay, RENO and Double Chooz experiments, have opened the path to the determination of the neutrino mass hierarchy. Indeed wheather the $v_{3}$ neutrino mass eigenstate is heavier or lighter than the $v_{1}$ and $v_{2}$ mass eigenstates is one of the remaining undetermined fundamental aspects of the Standard Model in the lepton sector. Mass hierarchy determination would have an impact in the quest of the neutrino nature (Dirac or Majorana mass terms) towards the formulation of a theory of flavour. The Jiangmen Underground Neutrino Observatory (JUNO) is a large liquid scintillator neutrino detector under construction in the south of China. Thanks to the large 20 kton active mass and unprecedented energy resolution $(3 \%$ at $1 \mathrm{MeV})$ it will allow to determine the neutrino mass hierarchy with good sensitivity and to precisely measure the neutrino mixing parameters, $\theta_{12}, \Delta m_{21}^{2}$ and $\Delta m_{e e}^{2}$ below the $1 \%$ level. The Reactor Experiment for Neutrino Oscillation (RENO-50) experiment is another medium distance reactor antineutrino experiment under construction in South Korea, with the same experimental challenges and goals. This paper will review the physics potential of medium baseline reactor antineutrino experiments, illustrate the technical characteristics of the JUNO and RENO-50 detectors, discuss the technological challenges and present the construction and R\&D status.

Neutrino Oscillation Workshop

4 - 11 September, 2016

Otranto (Lecce, Italy)

\footnotetext{
* Speaker.
} 


\section{Introduction}

The neutrino mixing angle $\theta_{13}$ has been measured with high precision by the Daya Bay [1], Reno [2] and Double Chooz [3] experiments; the sizable measured value has opened the way to measuring the neutrino mass hierarchy using antineutrinos from reactors. Three experimental approaches can be followed. The first possibility is to detect electron neutrino (antineutrino) appearance in a muon neutrino (antineutrino) beam using a long-baseline experiment. The approach is pursued by NovA in US and T2K in Japan. The disadvantage of these experiments is that the mass hierarchy information is highly dependent on the CP-violation phase. Another possibility is to detect neutrino and antineutrino interactions in a large atmospheric neutrino experiment like PINGU or ORCA, determining the mass hierarchy through the matter effects. These measurements are slightly affected by the uncertainties on the Earth density and atmospheric neutrino flux profiles, but strongly rely on the knowledge of some of the oscillation parameters: $\Delta m_{32}^{2}, \theta_{13}, \theta_{23}$, and $\delta_{C P}$ whose uncertainties and correlations may affect the interpretation of the measurements [4].

A third approach, that will be investigated in the present paper, is to detect neutrino oscillation at a medium-baseline from the source. Two large experiments have been proposed : the Jiangmen Underground Neutrino Observatory (JUNO) [5] is under construction in the South of China, while the Reactor Experiment for Neutrino Oscillation (RENO-50) [6] is in the R\&D stage in South Korea. Both experiments will detect electron antineutrino interactions in the main detector thanks to the inverse beta decay reaction $\bar{v}_{e}+p \rightarrow e^{+}+n$. The energy deposited by the positron annihilating in the liquid scintillator is proportional to the energy of the incoming antineutrinos. The time coincidence between the positron interaction and the subsequent neutron capture on protons allows to identify efficiently the neutrino interaction, even in the presence of uncorrelated background. The electron antineutrino survival probability can be written as

$$
P_{e e}=1-\sin ^{2} 2 \theta_{13} \cdot\left(\cos ^{2} \theta_{12} \sin ^{2} \Delta_{31}+\sin ^{2} \theta_{12} \sin ^{2} \Delta_{32}\right)-\sin ^{2} 2 \theta_{12} \cdot \cos ^{4} \theta_{13} \sin ^{2} \Delta_{12},
$$

where $\Delta_{i j}=\Delta m_{i j}^{2} L / 4 E_{v}$.

With the approximation $\Delta m_{32}^{2} \approx \Delta m_{31}^{2}$, it is possible to rewrite the survival probability and make explicit the mass hierarchy dependence in the formula

$$
\begin{aligned}
P_{e e}=1 & -\cos ^{4} \theta_{13} \sin ^{2} 2 \theta_{12} \sin ^{2} \Delta_{21}-\sin ^{2} \theta_{13} \sin ^{2}\left|\Delta_{31}\right| \\
& -\sin ^{2} \theta_{12} \sin ^{2} 2 \theta_{13} \sin ^{2} \Delta_{21} \cos 2\left|\Delta_{31}\right| \\
& \pm \frac{\sin ^{2} \theta_{12}}{2} \sin ^{2} 2 \theta_{13} \sin ^{2} 2 \Delta_{21} \sin ^{2} 2\left|\Delta_{31}\right|
\end{aligned}
$$

The sign flip in the last term is due to the neutrino mass hierarchy possibility: the direct hierarchy gives a positive contribution, while it is negative for the inverse mass ordering. The effect is small, but fortunately not negligible due to the relatively large value for $\theta_{13}$. The effect of the neutrino mass hierarchy on the electron antineutrino energy spectrum, measured at a medium-baseline of $53 \mathrm{~km}$, is shown in Figure 1 [7]. Thanks to the infinite energy resolution assumed for the reconstructed antineutrino energy (left plot), the two curves are clearly distinguishable. The right plot of the same Figure shows the reconstructed positron visible energy, having assumed a 3\% energy resolution at $1 \mathrm{MeV}$. The finite resolution of detectors tends to wash away the characteristic features of the ripples; if the resolution deteriorates further the peaks and throats of the spectrum would start 

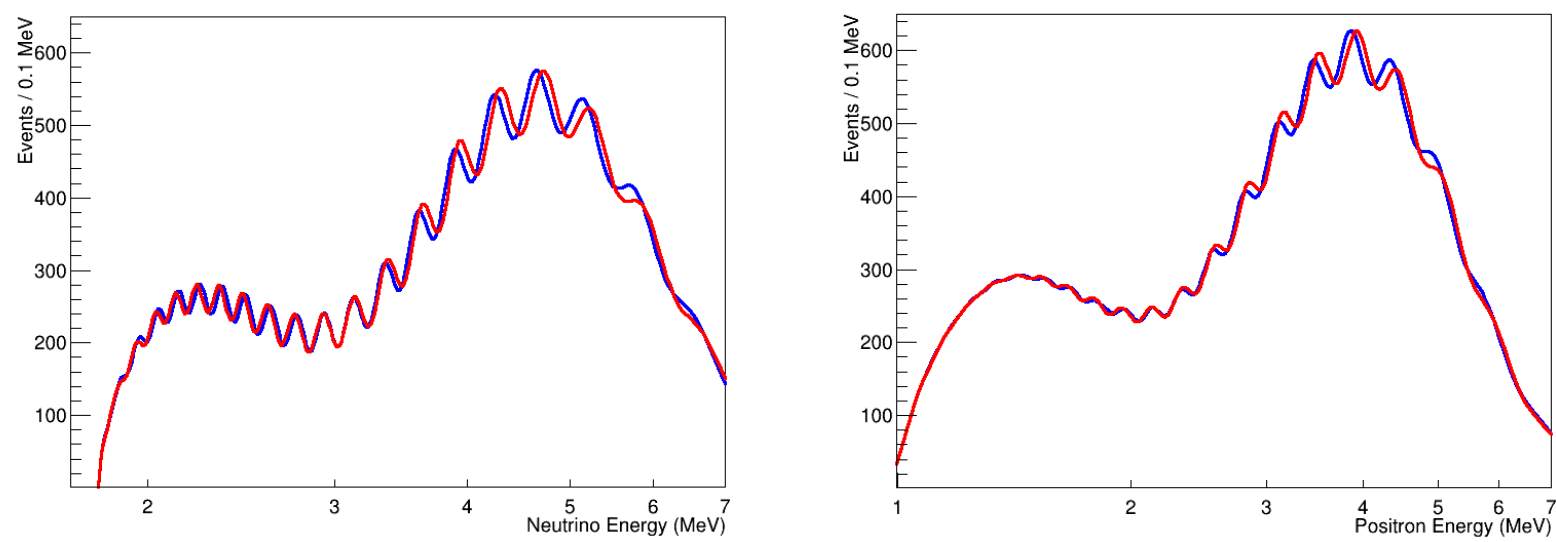

Figure 1: Left: electron antineutrino energy spectrum at a baseline of $53 \mathrm{~km}$ for an infinite energy resolution. Right: positron visible energy spectrum assuming a 3\%/ $\sqrt{E}$ detector energy resolution. Both normal (red) and inverted (blue) hierarchies are shown in the plots.

to disappear, making it very hard or almost impossible to unfold the neutrino mass hierarchy from the collected spectra.

Detailed studies have shown that assuming a large scintillator detector with a 20 kton mass, a $3 \%$ energy resolution and an exposure time of 6 years provide a $4 \sigma$ discrimination power (corresponding to a $\Delta \chi^{2}$ value of 16 between the true and wrong hierarchy hypothesis) [5]. Systematic effects would affect the result and worsen the discrimination power. The most important effect in this sense is the non-exactly equal baselines from the nuclear cores to the experiment, characterized by a spread of about $500 \mathrm{~m}$, which originates a loss that can be quantifies as a decrease of 4 of the $\Delta \chi^{2}$ indicator. Other adverse effects detrimental to the discovery power are the $1 \%$ shape uncertainty of the reactor spectrum, and the background uncertainty (rate $4.5 \%$, shape $0.35 \%$ ). All in all, these effects bring the discrimination power down to $\Delta \chi^{2}=10.4$, i.e. slightly more than $3 \sigma[5]$.

Beyond mass hierarchy and precision determination of the neutrino oscillation parameters, a large liquid scintillator detector can provide fundamental results on many topics in astroparticle physics, like supernova burst and diffuse supernova neutrinos, solar neutrinos, atmospheric neutrinos, geo-neutrinos, nucleon decay, indirect dark matter searches and a number of additional exotic searches. A reference to the rich physics program of JUNO can be found here [5].

\section{The JUNO Experiment}

JUNO is a large liquid scintillator detector with a 20 kton design mass and it will be located close to the Kaiping city, Jiangmen County, China. The detector will be constructed at a shallow depth, with an average overburden of $700 \mathrm{~m}$, to reduce the backgrounds induced by cosmogenic muons. The location of the experiment will be at about $53 \mathrm{~km}$ distance from the Yangiiang and Taishan nuclear power plants, where six and four nuclear cores are planned, respectively. According to the JUNO construction schedule, a total power of $26.6 \mathrm{GW}$ will be installed in 2020, and it will reach a $35.8 \mathrm{GW}$ total power in the following years. As can be seen from Figure 2 (left plot), 

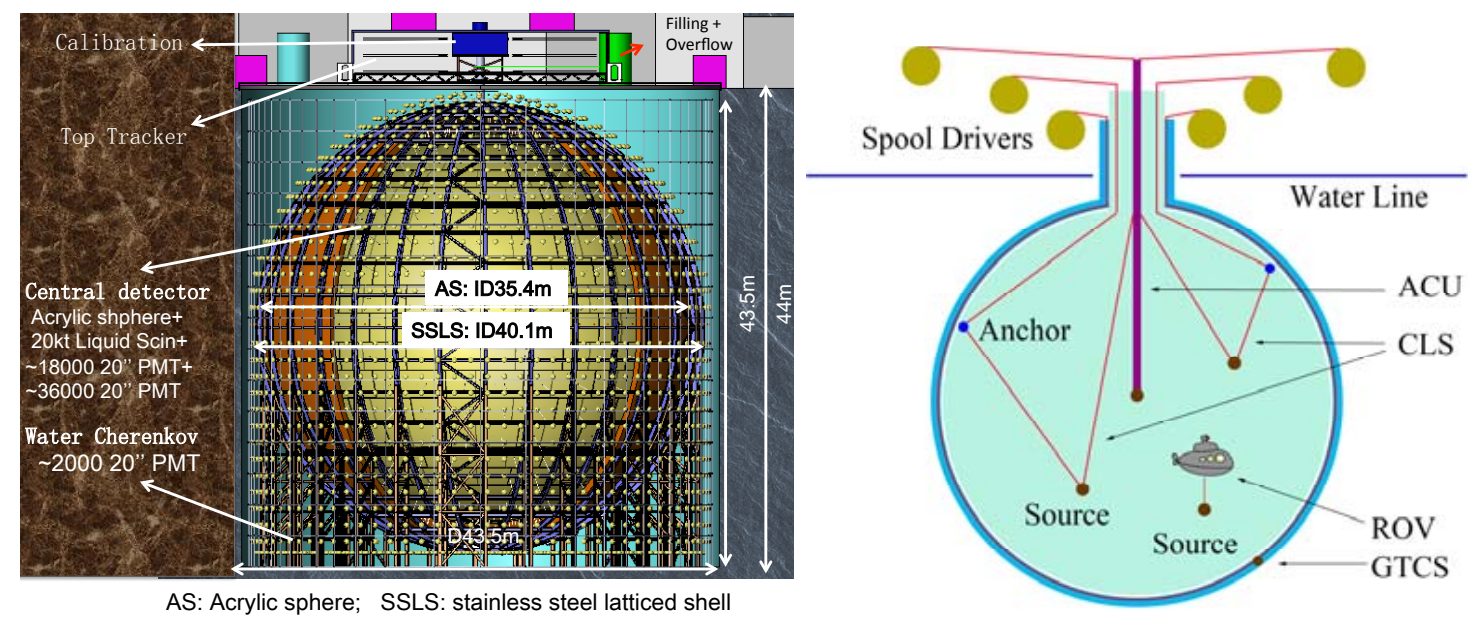

Figure 2: Left: JUNO experiment layout; details are discussed in the text. Right: JUNO Calibration system. The Automatic Calibration Unit (ACU), Cable Loop System (CLS), Guided Tube Calibration System (GTCS) and Remotely Operated Vehicle (ROV) are show in the picture.

the detector structure is quite simple: the liquid scintillator is contained in an acrylic sphere kept in position by a stainless steel truss. The central detector will be placed inside an active water pool that will act both as a Cherenkov muon veto and as shield against environmental radiation (gammas and neutrons) coming from the rock. An external veto detector, the plastic scintillator target tracker detector of the former OPERA [8] experiment at Gran Sasso [9] will be placed on top of the water pool.

As shown in Figure 2 (left plot), the liquid scintillator will be contained inside an acrylic vessel, $12 \mathrm{~cm}$ thick, with an inner diameter of $35.4 \mathrm{~m}$. The liquid scintillator will make use of LAB (Linear Alkyl Benzene) as solvent, with the scintillating PPO fluor (2.5-Diphenyloxazole) and a wavelength shifter (bis-MSB) diluted in LAB. The stainless steel truss will hold the inner vessel and support about 20000 large (20-inch) PMTs and 36000 small (3-inch) PMTs. The overall dimensions of the water pool, where the entire detector will be submerged, are $44 \mathrm{~m}$ high and with a diameter of $43.5 \mathrm{~m}$. In order to achieve the $3 \%$ energy resolution design goal at $1 \mathrm{MeV}$, about 18000 20-inch PMTs will face the inner part of the scintillator sphere. The remaining 2000 will instrument the water Cherenkov veto. Thanks to a high photocathode coverage (75\%), a mean light yield of about 1200 photoelectrons per $\mathrm{MeV}$ is expected, a number much larger than that the present generation neutrino liquid scintillator detectors ${ }^{1}$

Two types of large PMTs will be employed: about 15000 MCP-PMTs, a new development produced by North Night Vision Technology, will be used both for the inner detector and the water Cherenkov veto. The remaining 5000 PMTs will be commercially available large dynode PMTs (R12860-HQE), produced by Hamamatsu Photonics. In addition, JUNO will employ a dual calorimetry system, thanks to about 36000 3-inch PMTs which will be installed in the gaps between the large PMTs. Combining the two PMT systems, a total photocathode coverage of about $78 \%$ will be reached. The two system will work in synergy, providing redundancy in several of the

\footnotetext{
${ }^{1}$ as an example KamLAND has reached 250 pe/Mev, Daya Bay $160 \mathrm{pe} / \mathrm{MeV}$ and Borexino 500 pe/MeV.
} 
planned measurements of the experimental program. An high level of complementarity will be reached for what concerns time resolution and therefore spacial event reconstruction, improving the JUNO detection performances and enhancing the dynamic range and triggering capabilities.

A complete calibration system designed for multiple sources deployment is needed to reach the $3 \%$ at $1 \mathrm{MeV}$ design goal energy resolution and $1 \%$ energy scale uncertainty. As visible from Figure 2 (right plot), the experiment will make use of different tools for calibration: an Automatic Calibration Unit will allow to insert a radioactive sources along the detector central axis; a Cable Loop System will allow to position the source on a disc cutting the central detector from top to bottom. A Guide Tube Control System will allow to insert a radiation source along tubes fixed on the outer surface of the acrylic sphere and, finally, a Remotely Operated under-liquid-scintillator Vehicle will allow the source to be moved inside the liquid scintillator, reaching the whole central detector active area.

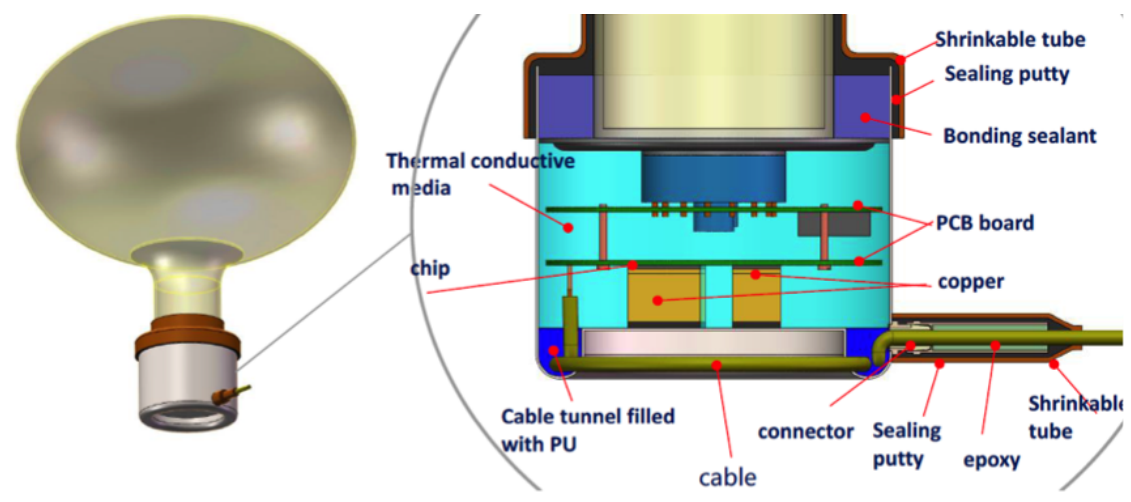

Figure 3: Left: JUNO large PMT with the base and readout electronics enclosed in the potting shell. Right: a detail of the potting shell; from top to bottom: base voltage divider, readout and power boards.

Another distinguishing feature of JUNO, with respect to previous large neutrino experiments, is that the readout electronics will be integrated in the PMT housing to reduce the number of cables and noise. A high readout accuracy is achived using a highly-integrated receiver chip which includes the analog to digital converter and the analog frontend with no external components used. After digitization, the digital part is achieved using an FPGA which compresses the data and includes timing information, as well as processing the signal for a fast trigger generation. A major feature in the processing scheme of the highly-integrated electronics is the vast configurability to select alternative modes and fine-tune operation parameters. The PMT biasing voltage will be generated very close to the PMT, thanks to a custom design high voltage module. All signals (synchronous and asynchronous) and power will be transported using standard CAT5 ethernet cables which will connect the single PMT to the dry electronics, in the counting rooms.

JUNO has been approved in China at the beginning of 2013. The ground breaking signaling the start-up of the excavation for the civil construction occurred at the beginning of 2015. So far, more than half of the slope tunnel ( $900 \mathrm{~m}$ out of $1340 \mathrm{~m}$ ) and about half of the vertical shaft ( $300 \mathrm{~m}$ out of $611 \mathrm{~m}$ ) have been excavated. The former will allow to bring the scintillator underground, and the latter will enable access of personnel and construction materials. The civil construction is foreseen to be completed by about middle of 2018 , including the large experimental hall. The 
preparation of the detector components, e.g. phototubes, acrylic panels, etc., has started in the current year 2016 and will encompass the whole 2017 and part of 2018, while the global on-site installation will be completed by the end of 2019. All this is in line to ensure scintillator filling and start-up of data taking within the targeted 2020 year.

\section{The RENO-50 Experiment}

The Reactor Experiment for Neutrino Oscillation, RENO-50, is a next generation underground experiment that will be constructed at about $50 \mathrm{~km}$ from the Hanbit nuclear power plant in South Korea. It will consist of about 18 kton of ultra low radioactivity liquid scintillator and 15000 large (20-inch) PMTs. Similarly to the JUNO detector, RENO-50 is expected to detect neutrinos from the reactors and from other natural (the Sun, the Earth and possible Supernova explosions) and artificial (J-PARC neutrino beam) neutrino sources. Similar performances, if compared to JUNO, are expected. The Collaboration is currently developing an R\&D program to test and develop the techniques needed to build and run the final experiment. Data taking is expected to start at the beginning of 2021 .

\section{Conclusion}

The quest for the determination of the neutrino mass hierarchy has started: several complementary approaches have been proposed and are pursued around the world. Next generation reactor neutrino experiments, with an unprecedented size, PMT coverage, light yield and energy resolution, have a high potentiality in determining the neutrino mass hierarchy using neutrino oscillations in vacuum. The JUNO Collaboration in China and the RENO-50 Collaboration in South Korea are rapidly progressing through the design, prototyping and construction phases and besides their main physics goal, they will also be able to precisely measure the neutrino oscillation parameters below the $1 \%$ level and attack a rich neutrino physics program. An exciting neutrino science program will start after the year 2020 when both experiments will be filled and, once commissioned, will start to collect data.

\section{References}

[1] F. .P. An et al, Measurement of electron antineutrino oscillation based on 1230 days of operation of the Daya Bay experiment, arXiv:1610.04802

[2] S. H. Seo et al, Spectral Measurement of the Electron Antineutrino Oscillation Amplitude and Frequency using 500 Live Days of RENO Data, arXiv:1610.04326

[3] Y. Abe at al, Measurement of $\theta_{13}$ in Double Chooz using neutron captures on hydrogen with novel background rejection techniques, JHEP01 (2016) 163, arXiv:1510.08937

[4] D. Franco et al, Mass hierarchy discrimination with atmospheric neutrinos in large volume ice/water Cherenkov detectors, JHEP04 (2013) 008

[5] F. An et al, J. Phys. G 43 (2016) 030401

[6] S. Kim, New results from RENO and prospects with RENO-50, arXiv:1412.2199 
[7] T. Adam et al, JUNO Conceptual Design Report, arXiv:1508.07166

[8] N. Agafonova et al., Phys. Rev. Lett. 115 (2015) 121802

[9] T. Adam et al, Nucl. Instr. Meth. A 577 (2007) 523 